\section{Modelling of the Plasma Boundary}

\author{
Ralf Schneider \\ Max-Planck-Institute für Plasma Physik, Garching, Germany
}

In any burning fusion plasma the auxiliary heating and alpha power (300 to $400 \mathrm{MW}$ in ITER) must be exhausted from the core plasma and delivered to surrounding structures. In the region of open field lines (outside the separatrix) the very strong parallel heat conduction leads to a very small power decay length and by this to extremely concentrated power deposition on the target plates (up to values close to the surface power densities of the sun of $50 \mathrm{MW} / \mathrm{m}^{2}$ ), which would be technologically impossible to handle without using additional mechanisms to spread the power over a wider area by, for example, hydrogen or impurity radiation losses to reach reasonable power loads below about $10 \mathrm{MW} / \mathrm{m}^{2}$.

The need for sufficient particle exhaust to avoid the extinguishing of the burning plasma by the helium ash also requires sufficient pumping capability (for ITER $5 \times 10^{20}$ alpha particles per second have to be pumped out). These technological problems of particle and power exhaust also have to be compatible with sufficiently good core confinement. This has motivated over the last few years an intensified effort to study both experimentally and theoretically the physics of the plasma boundary, especially that of the scrape-off layer where field-lines intersect specifically designed targets where the plasma is neutralized and the power transferred. To move this interaction zone away from the confined plasma a divertor is used where additional coils create a separatrix intersecting at the X-point.

Complex physics enters the plasma boundary description which represents a highly nonlinear problem. The plasma transport can be formulated as fluid equations with classical parallel transport and turbulent radial transport usually described semi-empirically by a Fick-like diffusion process with fitted transport coefficients. The very large parallel fluxes have large gradient lengths (typical of the order of connection lengths, eg 10 meters) compared to the usually smaller radial fluxes with gradient lengths of centimetres. Therefore, competition between these two processes already defines for the plasma transport a (at least) twodimensional situation. Each ion fluid is governed by a Navier-Stokes system of equations. Coupling with the plasma species occurs through ionization and recombination processes, interspecies friction, drifts, electric and thermal forces, and temperature equilibration. At the sheath entrance in front of the targets boundary conditions derived from kinetic models are necessary due to physics on the scale of the Debye length, where charge separation is possible. In the sheath a higher potential relative to the wall has to develop, because electrons are moving faster than ions due to their much smaller mass. The sheath reflects the slow electrons and enforces zero net current. The ions are accelerated to sound velocity (or greater) at the sheath entrance.

In addition, the interaction of ions with the surface creates neutrals by surface recombination and sputtering. These neutrals are self-consistently coupled with the plasma transport: ionization, charge exchange and recombination processes represent sink or source terms for the plasma which, on the other hand, determines the neutral transport.

Therefore, a coupled set of transport equations has to be solved both for the plasma (fluid equations) and the neutrals (either described by kinetic two or three dimensional Monte-Carlo or by fluid models). These plasma boundary codes were originally developed for tokamaks in two dimensions and have now been extended to three dimensions for stellarators. The different physics mechanisms involved require accurate molecular and atomic physics data-ionization, charge-exchange (CX) recombination, dissociation-surface reflection and sputtering data.

Even for the simplest case of a pure hydrogenic plasma the atomic physics defines different regimes. Experimentally, one can think of operating at a fixed input power and then doing a density ramp-up, passing through a sequence of different regimes. Because they are the result of atomic physics they are generic both for tokamak divertors and for stellarator (island) divertors.

In low recycling operation (divertor temperature $>20 \mathrm{eV}$, see figure 1) the hydrogen neutrals freely interpenetrate the plasma. The neutrals are in a kinetic regime where they do suffer typically up to one $\mathrm{CX}$ process before they are ionized ( $\mathrm{CX}$ and ionisation rates are similar, recombination is negligible). For the plasma conservation equations the neutral sources are unimportant: the plasma transport is dominated by very strong parallel electron heat conduction (proportional to $T^{5 / 2}$ ). The neutrals do not carry away any momentum through CX collisions with the plasma, therefore the parallel momentum equation reduces to pressure constancy along field lines with additional acceleration of the ions to sound speed at the sheath entrance. Density and temperature are constant along the fieldlines from midplane to the divertor, defining a very unfavourable regime at the target plates: one gets severe impurity sputtering problems due to the high divertor temperature and has no reduction of the target power load due to the lack of neutral energy losses.

Reducing the divertor temperature $(T<10 \mathrm{eV})$ by raising the midplane den-

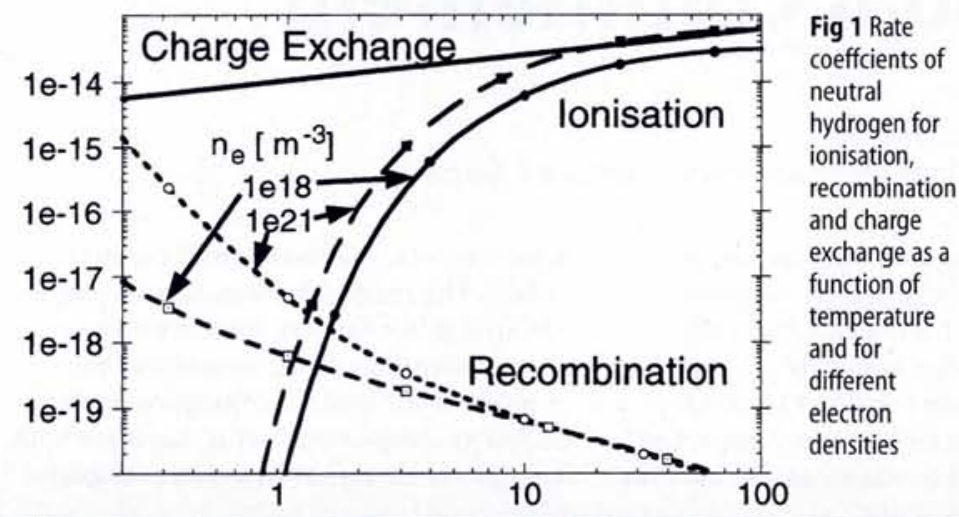



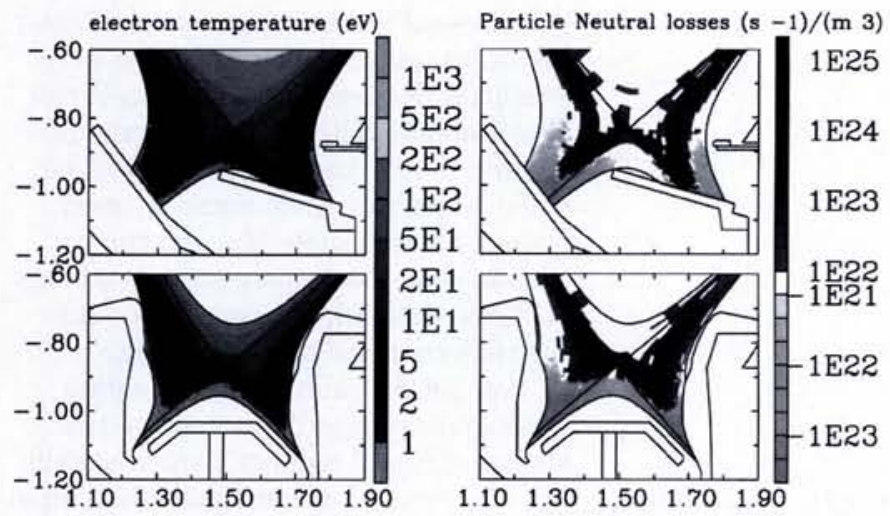

sity creates high recycling conditions: neutrals get diffusive in a random walk process, where they undergo for lower temperatures more and more $\mathrm{CX}$ collisions before they are ionized (CX larger than ionization rate coefficient). The ionization of neutrals is localized in the divertor and neutral sources get important especially for the continuity and energy equations. A recycling cycle is started, where the neutral particle sources drive ion fluxes and by thus enlarge again the neutral sources. One still gets pressure constancy along field lines, because the CX momentum losses are still negligible (a small neutral mean free path makes the divertor plasma fan opaque for neutrals). The enhanced ion divertor density gives a colder divertor with reduced physical sputtering, because the sputtering yields decrease towards lower temperature. However, this regime usually does not solve the power load problem, because the total neutral losses are limited by surface recombination of ions to a power load reduction factor of about 3 .

At rather low temperatures $(T<5 \mathrm{eV})$ the plasma detaches from the target. Momentum losses of the plasma get large by $\mathrm{CX}$ of neutrals with plasma ions and subsequent escape to the wall. A strong drop of the divertor power load is possible with the onset of strong volume recombination at temperatures of about $1 \mathrm{eV}$. The plasma recombines before reaching the target and creates a kind of virtual target. In this regime one also has very good pumping properties for helium, because recombination reduces the ion density in front of the plate and makes it easier for both the hydrogen and helium neutrals to escape out of the divertor plasma fan to the pumping ducts. The problem of this low-temperature regime is its control and stability; radiation instabilities can develop due to intrinsic impurities through a condensation instability.

Geometry strongly determines divertor effectiveness. As only a limited number of experimental configurations can be realized (money, time) validated $2 \mathrm{D}$ plasma boundary codes are used to derive optimized geometries satisfying the exhaust requirements and being compatible with core conditions.

A good example of this kind of engineering work motivated and directed by subtle physics is the change of the divertor in ASDEX Upgrade from a rather open configuration (DivI) to a strongly inclined geometry (DivII).

Comparing the contour plots of electron temperature and neutral particle sources by ionization and recombination for same upstream conditions one gets lower temperatures and a larger amount of volume recombination for DivII, especially in the high power flux region close to the separatrix. The strongly inclined target plates for DivII reflect neutrals preferentially towards this hot region and increase by this the neutral losses at the separatrix. Geometrically preferential detachment is provided and therefore a reduction of the power load at the separatrix (factor of 2 lower for DivII compared with DivI). As the outer SOL stays attached in DivII even when the separatrix zone is detached this is not changing the global detachment limit. Therefore, a maximum operational window is obtained. Additionally, better hydrogen and helium pumping is achieved.

Not yet satisfying is the need for an empirical description of the radial fluxes. Next step is to self-consistently couple turbulence codes which provide anomalous fluxes with boundary codes.

\section{Further reading}

C.S.Pitcher et al Plasma Phys. Controlled Fusion 39779 (1997)

D. Bohm The characteristics of electrical discharges in magnetic fields (Guthrie, Wakerling eds 1949)

W.- 0 . Hofer et al Physical Processes of the Interaction of Fusion Plasmas with Solids (Academic Press, London 1996)

\section{Quasi-Symmetries in Toroidal Confinement}

\section{Jürgen Nührenberg \\ Max-Planck-Institute für Plasma Physik, Greifswald, Germany}

Particles in toroidal plasmas with parameters typical of fusion performance have extremely long mean-free paths between collisions, typically a thousand times longer than the torus circumference. Therefore, an important aspect of toroidal plasma confinement is collision- less magnetic confinement of particle orbits. The magnetic moment of a particle, which is related to its fast gyromotion, is an adiabatic invariant and the motion of the centre of the gyro circle is called guiding-centre orbit. Together with energy conservation in a static magnetic field this invariant causes the reflection of particles which move into a region of increasing field strength for particles with sufficiently small velocities parallel to the magnetic field. This leads to the trapping of particles between regions of large field strength. In addition, guiding centre orbits experience drifts perpendicular to field lines due to field strength gradients and field line curvature. Collisions being rare, these two effects together will-in general, for arbitrary toroidal confinement regions-cause both, direct collisionless particle loss of high-energy, eg $\alpha$-particles and large diffusion coefficients of thermal particles. If the confinement domain exhibits a continuous symmetry-in a torus this is the 